\title{
Comparison of triglyceride/HDL-C ratio and triglyceride glucose index in identifying NAFLD in Chinese population: a cross-sectional study
}

\section{Fei Gao}

The Affiliated Hospital of Qingdao University

Hui Li

The Affiliated Hospital of Qingdao University

\section{Xinfeng Wang}

The Affiliated Hospital of Qingdao University

\section{Yingmei Zheng}

The Affiliated Hospital of Qingdao University

\section{Xuan Yang}

The Affiliated Hospital of Qingdao University

Jinyan Ren ( $\square$ renjinyan1983@163.com )

The Affiliated Hospital of Qingdao University https://orcid.org/0000-0002-5110-063X

\section{Research}

Keywords: TG/HDL-C ratio, TyG index, Nonalcoholic fatty liver disease, Insulin resistance

Posted Date: January 28th, 2021

DOl: https://doi.org/10.21203/rs.3.rs-155299/v1

License: (1) This work is licensed under a Creative Commons Attribution 4.0 International License. Read Full License 


\section{Abstract \\ Background}

Triglyceride (TG) to high-density lipoprotein cholesterol (HDL-C) ratio and triglyceride glucose index (TyG) have been recommended as surrogate markers for nonalcoholic fatty liver disease (NAFLD). In the present study, we aimed to compare the ability of TG/HDL-C, TyG and TyG-HDL-C, a new indicator deriving from TyG and HDL-C, in identifying NAFLD in Chinese population.

\section{Methods}

A total of 11,524 subjects who participated in a health checkup program were included. NAFLD was diagnosed by ultrasonography.

\section{Results}

The prevalence rates of NAFLD progressively increased across the quartiles of TG/HDL-C, TyG and TyGHDL-C ( $P$ for tend $<0.0001)$. After adjustment for confounding factors, the three indicators were all independently associated with the risk of NAFLD. The odds ratios (ORs) for NAFLD of the second to fourth TyG-HDL-C quartiles compared to the first quartile were 2.24 (1.92-2.62), 4.24 (3.64-4.94) and 8.96 (7.64-10.50), respectively. There was no difference in areas under the ROC curve (AUCs) of the three indicators in men, regardless of body weight. AUCs of TyG were significantly higher than thats of TG/HDL-C in overweight and obese women $(P<0.0001)$, with the optimal cut-off point of 8.56 (sensitivity $=74.4 \%$, specificity $=60.9 \%$ ). In normal or low body weight women, TyG-HDL-C, with a AUC of 0.827 (0.810-0.843), was better than the other two indictors, with AUCs of $0.813(0.796-0.830), 0.815(0.797-$ $0.831)$, respectively $(P=0.0015)$. The optimal cut-off point of TyG-HDL-C in normal or low body weight women was 6.8 (sensitivity $=78.1 \%$, specificity $=72.1 \%$ ).

\section{Conclusions}

TG/HDL-C is as effective as TyG in predicting NAFLD in Chinese men. TyG is superior to TG/HDL-C in identifying NFAFLD in Chinese overweight and obese women. While in Chinese normal and low weight women, TyG-HDL-C is a better predictor for NAFLD.

\section{Background}

Nonalcoholic fatty liver disease (NAFLD) ranges from simple hepatic steatosis to non-alcoholic steatohepatitis (NASH) and cirrhosis. NAFLD is the most common chronic liver disease worldwide, with an estimated global prevalence of $15-40 \%$ that continues to increase rapidly [1]. And in mainland of China, the prevalence is about $20.09 \%(17.95-22.31 \%)$ [2]. In recent years, several studies [3-6] have 
shown that NAFLD was also related to type 2 diabetes mellitus, abdominal obesity, dyslipidemia, hypertension and cardiovascular disease. Furthermore, the prediction for cardiovascular events was often independent of traditional cardiovascular risk factors [7]. Therefore, the early identification of NAFLD may be important for public health. There is a strong medical need of simple, noninvasive, cost-effective and reliable tools for predicting NAFLD.

Recently, the triglyceride and glucose index (TyG) [8-10] and triglyceride to high-density lipoprotein cholesterol (TG/HDL-C) ratio [11-14] have been recommended as an effective biomarker to identify NAFLD. To the best of our knowledge, there is no comparative study of TyG and TG/HDL-C in predicting NAFLD. Moreover, we speculated that whether TyG-HDL-C, concluding triglyceride (TG), fasting plasma glucose (FPG) and high-density lipoprotein cholesterol (HDL-C), can be better in predicting NAFLD.

Therefore, in the present study we sought to compare the ability of the TyG index, TG/HDL ratio and TyGHDL-C in detectig NAFLD in Chinese adults with different sex and weight.

\section{Methods}

\section{Subjects}

The study population was recruited from adults who underwent health checkups at the Affiliated Hospital of Qingdao University between January 2018 and December 2018. Subjects with an alcohol intake > $140 \mathrm{~g} /$ week for men and $70 \mathrm{~g} /$ week for women, a history of viral hepatitis, auto-immune hepatitis or other forms of chronic liver disease were excluded from the study. Finally, a total of 11,524 subjects were included in the final analysis, consisting of 6,326 men and 5,198 women.

Our study was approved by institutional review board of the Affiliated Hospital of Qingdao University, and the informed consent requirement was exempted because of our retrospective estimation of unidentified database.

\section{Clinical measurements}

Physical examination was performed and anthropometry was obtained comprised of weight, height, systolic and diastolic blood pressure (SBP, DBP). Body mass index (BMI) was calculated as body weight $(\mathrm{kg})$ divided by body height squared $\left(\mathrm{m}^{2}\right)$. According to the criteria for Chinese people, normal and low weight was defined as $\mathrm{BMI}<24 \mathrm{~kg} / \mathrm{m}^{2}$, overweight as $\mathrm{BMI} \geq 24$ and $<28 \mathrm{~kg} / \mathrm{m}^{2}$, and obesity as $\mathrm{BMI} \geq 28$ $\mathrm{kg} / \mathrm{m}^{2}$. Fasting blood samples were collected after at least $10 \mathrm{~h}$ overnight. Triglycerides (TG), total cholesterol (TC), low-density lipoprotein cholesterol (LDL-C), high-density lipoprotein cholesterol (HDL-C), FPG, serum uric acid (SUA) and homocysteine (HCY) were measured using an autoanalyzer (Beckman, Palo Alto, CA). The TyG index was calculated as established formulas, TyG $=\mathrm{Ln}[\mathrm{TG}(\mathrm{mg} / \mathrm{dL}) \times \mathrm{FPG}$ (mg/dL)/2] [15]. TyG-HDL-C was calculated by subtracting HDL-C(mmol/L) from TyG.

\section{Diagnosis of NAFLD}


NAFLD was diagnosed according to the guidelines of NAFLD issued by the Chinese National Consensus Workshop on Nonalcoholic Fatty Liver Disease [16]. The diagnosis of NAFLD was based on ultrasonography findings of hepatic steatosis associated with characteristic echo patterns, including ultrasound beam attenuation, diffuse hyperechogenicity of liver, and poor visualization of intrahepatic structures, using a Toshiba Nemio 20 sonography machine with a 3.5-MHz probe (Toshiba, Tokyo, Japan).

\section{Statistical analysis}

Continuous variables were presented as median (interquartile range) because of their skew distribution, and categorical variables were displayed as percentages (\%). The significance of differences between groups was evaluated by Mann-Whitney $U$ test for continuous variables and chi-square test for categorical variables. Logistic regression was used to evaluate the association between TG/HDL-C, TyG, TyG-HDL-C and NAFLD. Four models were applied: model 1 was unadjusted. Model 2 was adjusted for age, sex and BMI. Model 3 was adjusted for all variables in model 2 plus SBP, SUA and Hcy. Model 4 was adjusted for all variables in model 3 plus HDL-C for TyG quartiles or plus FPG for TG/HDL-C quartiles. Receiver operator characteristic (ROC) curve analyses were performed to assess the abilities of the three indicators to detect NAFLD. $Z$ test was used for comparing the differences between the areas under the curve (AUCs). $P<0.05$ was considered statistically significant. SPSS for Windows version 23.0 (IBM Co., Armonk, NY, USA) and MedCalc (MedCalc software, Olstead, Belgium) were used for statistical analyses in the present study.

\section{Results}

\section{Clinical characteristics of the study population}

A total of 11,524 subjects were included in the present study. The mean age of the population was 55.7 $( \pm 11.5)$ years and mean BMI $25.8( \pm 3.5) \mathrm{kg} / \mathrm{m}^{2}$. There were 4,843 participants diagnosed as NAFLD by liver ultrasonic examination, with a prevalence of $42 \%$. In comparison with subjects without NAFLD, subjects with NAFLD were more likely to be male, and had higher levels of BMI, SBP, DBP, FPG, TG, TC, LDL-C, UA, HCY, TG/HDL-C ratio, TyG and TyG-HDL-C $(P<0.0001)$. In contrast, HDL-C was significantly reduced in the NAFLD group. Notably, the mean age of women with NAFLD was older than that of without NAFLD, while the mean age of men with NAFLD was younger than that of without NAFLD (Table 1). 
Table 1

Clinical Characteristics of the Study Subjects Based on NAFLD Status

\begin{tabular}{|c|c|c|c|c|}
\hline & group & Non-NAFLD & NAFLD & $P$ value \\
\hline Total, N (\%) & total & $6681(58 \%)$ & $4843(42 \%)$ & \\
\hline Men, N (\%) & total & $3079(46.1 \%)$ & $3247(67 \%)$ & $<0.0001$ \\
\hline \multirow[t]{3}{*}{ Age, years } & men & $52(44-61)$ & $49(42-56)$ & $<0.0001$ \\
\hline & women & $52(44-59)$ & $56(51-63)$ & $<0.0001$ \\
\hline & total & $52(44-60)$ & $54(45-59)$ & 0.426 \\
\hline \multirow[t]{3}{*}{$\mathrm{BMI}, \mathrm{kg} / \mathrm{m} 2$} & men & $24.73(22.94-26.58)$ & $27.71(25.95-29.76)$ & $<0.0001$ \\
\hline & women & $23.73(21.78-25.91)$ & $27.27(25.28-29.52)$ & $<0.0001$ \\
\hline & total & $24.24(22.31-26.26)$ & $27.58(25.71-29.72)$ & $<0.0001$ \\
\hline \multirow[t]{3}{*}{ SBP, mmHg } & men & 127(117-139) & $131(121-142)$ & $<0.0001$ \\
\hline & women & $122(111-136)$ & $135(122-150)$ & $<0.0001$ \\
\hline & total & $125(114-138)$ & $132(121-145)$ & $<0.0001$ \\
\hline \multirow[t]{3}{*}{$\mathrm{DBP}, \mathrm{mmHg}$} & men & $79(72-87)$ & $84(76-92)$ & $<0.0001$ \\
\hline & women & $73(65-81)$ & $80(72-88)$ & $<0.0001$ \\
\hline & total & $76(68-85)$ & $83(75-90)$ & $<0.0001$ \\
\hline \multirow[t]{3}{*}{$\mathrm{FPG}, \mathrm{mmol} / \mathrm{L}$} & men & $5.01(4.69-5.43)$ & $5.30(4.88-5.90)$ & $<0.0001$ \\
\hline & women & $4.90(4.59-5.26)$ & $5.35(4.92-5.99)$ & $<0.0001$ \\
\hline & total & $4.96(4.63-5.34)$ & $5.31(5.90-5.93)$ & $<0.0001$ \\
\hline \multirow[t]{3}{*}{$\mathrm{TG}, \mathrm{mmol} / \mathrm{L}$} & men & $1.12(0.81-1.58)$ & $1.81(1.26-2.62)$ & $<0.0001$ \\
\hline & women & $0.97(0.71-1.36)$ & $1.64(1.19-2.29)$ & $<0.0001$ \\
\hline & total & $1.04(0.75-1.47)$ & $1.75(1.23-2.50)$ & $<0.0001$ \\
\hline \multirow[t]{3}{*}{$\mathrm{TC}, \mathrm{mmol} / \mathrm{L}$} & men & $5.00(4.41-5.64)$ & $5.22(4.62-5.88)$ & $<0.0001$ \\
\hline & women & $5.14(4.52-5.85)$ & $5.46(4.81-6.19)$ & $<0.0001$ \\
\hline & total & $5.08(4.46-5.75)$ & $5.29(4.69-5.98)$ & $<0.0001$ \\
\hline \multirow[t]{2}{*}{$\mathrm{HDL}-\mathrm{C}, \mathrm{mmol} / \mathrm{L}$} & men & $1.41(1.22-1.63)$ & $1.25(1.10-1.43)$ & $<0.0001$ \\
\hline & women & $1.62(1.41-1.86)$ & $1.40(1.22-1.59)$ & $<0.0001$ \\
\hline
\end{tabular}

Data are presented as median (interquartile range) or percentage 


\begin{tabular}{|c|c|c|c|c|}
\hline & group & Non-NAFLD & NAFLD & $P$ value \\
\hline & total & $1.52(1.31-1.77)$ & $1.30(1.13-1.49)$ & $<0.0001$ \\
\hline \multirow[t]{3}{*}{ LDL-C, mmol/L } & men & $2.96(2.48-3.46)$ & $3.19(2.67-3.69)$ & $<0.0001$ \\
\hline & women & $2.91(2.43-3.49)$ & $3.30(2.78-3.85)$ & $<0.0001$ \\
\hline & total & $2.93(2.45-3.48)$ & $3.22(2.71-3.74)$ & $<0.0001$ \\
\hline \multirow[t]{3}{*}{ SUA, mmol/L } & men & $359(313-407)$ & $402(350-458)$ & $<0.0001$ \\
\hline & women & $268(233-306)$ & $312(273-356)$ & $<0.0001$ \\
\hline & total & $305(255-365)$ & $372(313-433)$ & $<0.0001$ \\
\hline \multirow[t]{3}{*}{$\mathrm{HCY}, \mathrm{mmol} / \mathrm{L}$} & men & $11.80(10.00-14.20)$ & $11.80(10.18-14.05)$ & 0.885 \\
\hline & women & $9.30(7.91-11.04)$ & $9.83(8.44-11.59)$ & $<0.0001$ \\
\hline & total & $10.40(8.70-12.60)$ & $11.12(9.46-13.25)$ & $<0.0001$ \\
\hline \multirow[t]{3}{*}{ TG/HDL-C } & men & $0.79(0.53-1.20)$ & $1.43(0.95-2.21)$ & $<0.0001$ \\
\hline & women & $0.60(0.40-0.91)$ & $1.15(0.79-1.72)$ & $<0.0001$ \\
\hline & total & $0.68(0.45-1.05)$ & $1.34(0.88-2.06)$ & $<0.0001$ \\
\hline \multirow[t]{3}{*}{ TyG } & men & $8.43(8.08-8.82)$ & $8.97(8.59-9.40)$ & $<0.0001$ \\
\hline & women & $8.27(7.92-8.63)$ & $8.88(8.54-9.29)$ & $<0.0001$ \\
\hline & total & $8.34(7.99-8.72)$ & $8.94(8.57-9.37)$ & $<0.0001$ \\
\hline \multirow[t]{3}{*}{ TyG-HDL-C } & men & $7.00(6.54-7.49)$ & $7.68(7.24-8.20)$ & $<0.0001$ \\
\hline & women & $6.62(6.11-7.12)$ & 7.46(7.00-7.97) & $<0.0001$ \\
\hline & total & $6.80(6.29-7.31)$ & $7.62(7.16-8.13)$ & $<0.0001$ \\
\hline
\end{tabular}

Data are presented as median (interquartile range) or percentage

\section{Comparison of the associations of TG/HDL-C, TyG and TyG- HDL-C with NAFLD risk}

The prevalence of NAFLD was all significantly increased along with the TG/HDL-C, TyG and TyG-HDL-C quartiles ( $P$ for trend $<0.0001$, Fig. 1). The phenomenon is more obvious in TyG-HDL-C quartiles. The prevalence of the highest TyG-HDL-C quartile was $73.7 \%$, which showed a 6.8 -fold increased with the ones in the lowest quartile. While the prevalence of the fourth quartile of TG/HDL-C and TyG rose 6.2 times and 6.3 times, respectively, compared with that of ones in lowest quartile.

We also observed a significant trend of increasing odds ratio for NAFLD with increasing levels of TG/HDL-C, TyG and TyG-HDL-C in Table 2 ( $P$ for trend $<0.0001$ ). The crude ORs for NAFLD (model 1$)$ of 
the second to fourth TyG-HDL-C quartiles compared to the first quartile (3.8 (3.3-4.3), 9.1 (7.9-10.5), and 23.2 (20.1-26.8), respectively) were greater than corresponding figures of TyG quartiles and TG/HDL-C quartiles. After adjustment for age, sex and BMI (model 2), and further for SBP, SUA and HCY (model 3), the odds ratios (ORs) for NAFLD remained progressively increased across quartiles of TG/HDL-C, TyG and TyG-HDL-C. The ORs for NAFLD of the second to fourth TyG-HDL-C quartiles compared to the first quartile were 2.24 (1.92-2.62), 4.24 (3.64-4.94) and 8.96 (7.64-10.50), respectively. Finally, the associations persisted after additional adjustment for HDL-C in TyG quartiles and FPG in TG/HDL-C quartiles (model 4). 
Table 2

Odds ratios for NAFLD in different quartiles of TyG index or ALT

\begin{tabular}{|c|c|c|c|c|c|}
\hline & & Model1 & Model2 & Model3 & Model4 \\
\hline \multirow{4}{*}{$\begin{array}{l}\text { TG/HDL- } \\
\mathrm{C}\end{array}$} & Q1(Reference) & 1 & 1 & 1 & 1 \\
\hline & $\begin{array}{l}\text { Q2(OR, 95\% } \\
\mathrm{Cl})\end{array}$ & $3.71(3.23-4.26)$ & $\begin{array}{l}2.51(2.16- \\
2.91)\end{array}$ & $\begin{array}{l}2.37(2.03- \\
2.75)\end{array}$ & $\begin{array}{l}2.27(1.95- \\
2.64)\end{array}$ \\
\hline & $\begin{array}{l}\text { Q3(OR, 95\% } \\
\mathrm{Cl})\end{array}$ & $7.89(6.89-9.03)$ & $\begin{array}{l}4.28(3.69- \\
4.96)\end{array}$ & $\begin{array}{l}3.91(3.36- \\
4.54)\end{array}$ & $\begin{array}{l}3.69(3.17- \\
4.29)\end{array}$ \\
\hline & $\begin{array}{l}\text { Q4(OR, 95\% } \\
\mathrm{Cl})\end{array}$ & $\begin{array}{l}20.06(17.43- \\
23.07)\end{array}$ & $\begin{array}{l}9.45(8.11- \\
11.02)\end{array}$ & $\begin{array}{l}8.04(6.88- \\
9.39)\end{array}$ & $\begin{array}{l}7.18(6.14- \\
8.41)\end{array}$ \\
\hline \multirow[t]{4}{*}{ TyG } & Q1(Reference) & 1 & 1 & 1 & 1 \\
\hline & $\begin{array}{l}\text { Q2(OR, 95\% } \\
\mathrm{Cl})\end{array}$ & $3.55(3.10-4.08)$ & $\begin{array}{l}2.45(2.10- \\
2.85)\end{array}$ & $\begin{array}{l}2.30(1.98- \\
2.68)\end{array}$ & $\begin{array}{l}2.19(1.88- \\
2.56)\end{array}$ \\
\hline & $\begin{array}{l}\text { Q3(OR, 95\% } \\
\mathrm{Cl})\end{array}$ & $7.83(6.84-8.96)$ & $\begin{array}{l}4.51(3.89- \\
5.23)\end{array}$ & $\begin{array}{l}4.08(3.51- \\
4.74)\end{array}$ & $\begin{array}{l}3.74(3.22- \\
4.36)\end{array}$ \\
\hline & $\begin{array}{l}\text { Q4(OR, 95\% } \\
\mathrm{Cl})\end{array}$ & $\begin{array}{l}20.65(17.95- \\
23.77)\end{array}$ & $\begin{array}{l}10.75(9.22- \\
12.54)\end{array}$ & $\begin{array}{l}9.26(7.92- \\
10.82)\end{array}$ & $\begin{array}{l}7.99(6.79- \\
9.39)\end{array}$ \\
\hline \multirow{4}{*}{$\begin{array}{l}\text { TyG- } \\
\text { HDL-C }\end{array}$} & Q1(Reference) & 1 & 1 & & \\
\hline & $\begin{array}{l}\text { Q2(OR, 95\% } \\
\mathrm{Cl})\end{array}$ & $3.75(3.26-4.32)$ & $\begin{array}{l}2.37(2.03- \\
2.77)\end{array}$ & $\begin{array}{l}2.24(1.92- \\
2.62)\end{array}$ & \\
\hline & $\begin{array}{l}\mathrm{Q} 3(\mathrm{OR}, 95 \% \\
\mathrm{Cl})\end{array}$ & $\begin{array}{l}9.10(7.92- \\
10.45)\end{array}$ & $\begin{array}{l}4.67(4.01- \\
5.42)\end{array}$ & $\begin{array}{l}4.24(3.64- \\
4.94)\end{array}$ & \\
\hline & $\begin{array}{l}\mathrm{Q} 4(\mathrm{OR}, 95 \% \\
\mathrm{Cl})\end{array}$ & $\begin{array}{l}23.16(20.06- \\
26.75)\end{array}$ & $\begin{array}{l}10.41(8.89- \\
12.17)\end{array}$ & $\begin{array}{l}8.96(7.64- \\
10.50)\end{array}$ & \\
\hline \multicolumn{6}{|c|}{ Model 1 was unadjusted. } \\
\hline \multicolumn{6}{|c|}{ Model 2 was adjusted for age, sex and BMI. } \\
\hline \multicolumn{6}{|c|}{ Model 3 was adjusted for all variables in model 2 plus SBP, SUA and HCY. } \\
\hline
\end{tabular}

\section{Diagnostic accuracy of TG/HDL, TyG and TyG-HDL-C for NAFLD}

To compare the ability of TG/HDL-C, TyG and TyG-HDL-C for detecting NAFLD, subjects were further grouped by sex and weight, and the ROC curves were analyzed. As shown in Table 3, with the decrease of $\mathrm{BMI}$, the areas under the ROC curve (AUCs) of the three indicators became larger, except for TyG in men. 
There was no difference in AUCs of the three indicators in men, regardless of body weight. Furthermore, the optimal cut-off point of TG/HDL-C was 1.02 in men, with a sensitivity of $70.9 \%$ and specificity of 66.4 $\%$.

Table 3

Comparison of AUCs $(95 \%$ Cl) of evaluated parameters for NAFLD in subjects categorized by sex and weight

\begin{tabular}{|c|c|c|c|c|c|c|}
\hline \multirow[t]{2}{*}{ Groups } & \multicolumn{2}{|c|}{ TG/HDL-C } & \multicolumn{2}{|l|}{ TyG } & \multicolumn{2}{|c|}{ TyG-HDL-C } \\
\hline & $\begin{array}{l}\text { AUROC } \\
(95 \% \mathrm{Cl})\end{array}$ & $P$ & $\begin{array}{l}\text { AUROC } \\
(95 \% \mathrm{Cl})\end{array}$ & $P$ & $\begin{array}{l}\text { AUROC } \\
\text { ( } 95 \% \mathrm{Cl})\end{array}$ & $P$ \\
\hline \multicolumn{7}{|l|}{ Men } \\
\hline Obesity & $\begin{array}{l}0.641 \\
(0.619- \\
0.664)\end{array}$ & $\begin{array}{l}<.0001 \\
0 .\end{array}$ & $\begin{array}{l}0.654 \\
(0.631- \\
0.676)\end{array}$ & $\dot{0} .0001$ & $\begin{array}{l}0.644 \\
(0.621- \\
0.666)\end{array}$ & $\begin{array}{l}< \\
0.0001\end{array}$ \\
\hline Overweight & $\begin{array}{l}0.703 \\
(0.687- \\
0.719)\end{array}$ & $\begin{array}{l}<.0001 \\
0.0\end{array}$ & $\begin{array}{l}0.704 \\
(0.687- \\
0.720)\end{array}$ & $\begin{array}{l}< \\
0.0001\end{array}$ & $\begin{array}{l}0.706 \\
(0.689- \\
0.722)\end{array}$ & $\begin{array}{l}< \\
0.0001\end{array}$ \\
\hline $\begin{array}{l}\text { Normal and } \\
\text { low weight }\end{array}$ & $\begin{array}{l}0.756 \\
(0.733- \\
0.778)\end{array}$ & $\begin{array}{l}<.0001 \\
0.0\end{array}$ & $\begin{array}{l}0.769 \\
(0.746- \\
0.791)\end{array}$ & $\begin{array}{l}< \\
0.0001\end{array}$ & $\begin{array}{l}0.753 \\
(0.729- \\
0.775)\end{array}$ & $\begin{array}{l}< \\
0.0001\end{array}$ \\
\hline \multicolumn{7}{|l|}{ Women } \\
\hline Obesity & $\begin{array}{l}0.718 \\
(0.688- \\
0.745)\end{array}$ & $\begin{array}{l}< \\
0.0001\end{array}$ & $\begin{array}{l}0.751 \\
(0.723- \\
0.778)^{a}\end{array}$ & $\begin{array}{l}< \\
0.0001\end{array}$ & $\begin{array}{l}0.739 \\
(0.710- \\
0.766)^{a}\end{array}$ & $\begin{array}{l}<.0001 \\
0 .\end{array}$ \\
\hline Overweight & $\begin{array}{l}0.725 \\
(0.705- \\
0.744)\end{array}$ & $\dot{0} .0001$ & $\begin{array}{l}0.741 \\
(0.722- \\
0.759)^{a}\end{array}$ & $\begin{array}{l}< \\
0.0001\end{array}$ & $\begin{array}{l}0.743 \\
(0.724- \\
0.762)^{a}\end{array}$ & $\begin{array}{l}< \\
0.0001\end{array}$ \\
\hline $\begin{array}{l}\text { Normal and } \\
\text { low weight }\end{array}$ & $\begin{array}{l}0.813 \\
(0.796- \\
0.830)\end{array}$ & $\dot{0.0001}$ & $\begin{array}{l}0.815 \\
(0.797- \\
0.831)\end{array}$ & $\dot{0} .0001$ & $\begin{array}{l}0.827 \\
(0.810- \\
0.843)^{b}\end{array}$ & $\begin{array}{l}< \\
0.0001\end{array}$ \\
\hline
\end{tabular}

Comparison of AUCs using $Z$ test. ${ }^{a}$ means a significant difference compared with that of TG/HDL-C in the same weight group. ${ }^{b}$ means a significant difference compared with that of TyG in the same weight group.

In contrast, the AUCs of TyG were significantly higher than thats of TG/HDL-C in overweight and obese women, while similar to thats of TyG-HDL-C. The optimal cut-off point of TyG for NAFLD of overweight and obese women was 8.56 , with a sensitivity of $74.4 \%$ and specificity of $60.9 \%$. In normal or low body weight women, the AUCs of TG/HDL-C and TyG had no difference, while TyG-HDL-C was better than the former two. The optimal cut-off point of TyG-HDL-C in normal or low body weight women was 6.8 , with a sensitivity of $78.1 \%$ and specificity of $72.1 \%$ (Fig. 2). 


\section{Discussion}

In the present study,we observed TG/HDL-C, TyG and TyG-HDL-C were all closely and positively related to risk of NAFLD, after adjustment for potential confounders. We also demonstrated that, in men, there was no difference in the effectiveness of TG/HDL-C, TyG and TyG-HDL-C for identifying NAFLD, regardless of body weight. In contrast, TyG was superior to TG/HDL-C for predicting NAFLD in overweight and obese women, while not different from TyG-HDL-C. In women with normal or low body weight, TyG and TG/HDL$C$ had no difference in identification for NAFLD, while TyG-HDL-C was better than the former two. The optimal cut-off point of TyG-HDL-C for NAFLD in normal and low body weight women was 6.8 . The optimal cut-off point of TyG for NAFLD in overweight and obese women was 8.56. Compared with the other two indicators, TG/HDL-C is more suitable for predicting NAFLD in Chinese men for its simple calculation, with the cut-off point of 1.02, although there was no difference in effectiveness.

In recent years, a growing body of evidence has shown an association between TG/HDL-C and NAFLD, as well as TyG and NAFLD. Two cohort studies $[12,14]$ showed that higher TG/HDL-C was strongly associated with increased risk of NAFLD. Similarly, in a cross-sectional study [11] involving 18,061 participants, TG/HDL-C was independently related with NAFLD in apparently healthy individuals and was recommended as a surrogate for NAFLD. Several cross-sectional studies [8-10] aslo revealed a strong and positive association between TyG and NAFLD, and TyG was effective to screen NAFLD. Similarly, in a nine-year cohort study [17], the researchers found that TyG could predict the risk of NAFLD in the next few years. Consistently, our present study also revealed a close relationship between TG/HDL-C and NAFLD, as well as TyG and NAFLD. Furthermore, both TG/HDL-C and TyG were effective in detecting NAFLD.

The mechanisms associated with the onset of NAFLD remain poorly understood. The current evidence [18] suggests a "multiple-hit" hypothesis including insulin resistance (IR), hormones secreted from the adipose tissue, nutritional factors, gut microbiota and genetic and epigenetic factors, among which IR plays an important role. Moreover, IR has been found to be associated with the development of NAFLD $[19,20]$. Besides,the severity of IR was also positively related to the progress and prognosis of NAFLD [21]. On the other hand, TG/HDL-C [22-25] and TyG [10, 26-29] were both found to be closely associated with insulin resistance in different populations and have been proposed as markers of insulin resistance. Thus, IR may be the mechanism underlying the link between TG/HDL-C, TyG and NAFLD.

Previous study by Er et al. [30] showed that TyG was more efficient than TG/HDL-C for identifying insulin resistance. Another study by Babic et al. [31] showed TG/HDL-C might be a useful predictor of glycemic control in normal weight, and TyG index in overweight and obese patients with diabetes mellitus type 2 . The comparison of TG/HDL-C and TyG in predicting NAFLD was not investigated in previous studies. We created a new indicator, TyG-HDL-C, deriving from three variables highly correlated with IR. Besides, BMI is an important factor affected TyG efficacy for identifying NAFLD [8]. Thus, our present study compared the ability of TG/HDL-C, TyG and TyG-HDL-C in predicting NAFLD in different sex and weight groups. Our study found with the decrease of BMI, all of the three indicators were more closely related with NAFLD. There was no difference in the ability of TG/HDL-C, TyG and TyG-HDL-C for identifying NAFLD, regardless 
of body weight. Compared with the other two indicators, TG/HDL-C, with a AUC of 0.746 (0.735-0.757), is more suitable for predicting NAFLD in Chinese men for its simple calculation, although there was no difference in effectiveness. And the optimal cut-off point was 1.02, with a sensitivity of $70.9 \%$ and specificity of $66.4 \%$. In contrast, TyG was superior to TG/HDL-C for predicting NAFLD in overweight and obese women, while not different from TyG-HDL-C. The optimal cut-off point of TyG for diagnosing NAFLD in overweight and obese women was 8.56, with a AUC of 0.748 (0.732-0.763), a sensitivity of $74.4 \%$ and specificity of $60.9 \%$. In women with normal or low body weight, TyG and TG/HDL-C had no difference in identification for NAFLD, while TyG-HDL-C was better than the former two. The optimal cutoff point of TyG-HDL-C for NAFLD in normal or low body weight women was 6.8, with a AUC of 0.827 (0.810-0.843), a sensitivity of $78.1 \%$ and specificity of $72.1 \%$.

The differences of TyG and TG/HDL-C in diagnostic efficacy between men and women may be caused by the different distribution of HDL-C in gender, which had dose-response relationship with IR and the risk of NAFLD [32-34]. In our study, the level of HDL-C in men was lower than that in women (men, $1.36 \pm$ $0.32 \mathrm{mmol} / \mathrm{L}$ vs women, $1.58 \pm 0.35 \mathrm{mmol} / \mathrm{L}, P<0.0001)$, and the prevalence of low HDL-C in men was significantly higher than that in women (men, $11.8 \%$ vs women, $3.9 \%, P<0.0001$ ). A nationally representative survey [35] of 163,641 Chinese adults also found low HDL-C and high TG were the primary forms of dyslipidemia, and the prevalence of low HDL-C in men was much higher than that in women. In the Chinese population, low HDL-C mainly affects men. This may be the reason why TG/HDL-C is weaker than TyG in identifying NAFLD, while in men, TG/HDL-C is as effective as TyG. We also found a phenomenon that the mean age of women with NAFLD was older than that of without NAFLD, while the mean age of men with NAFLD was younger than that of without NAFLD. This may be related to different exercise and eating habits of men in different ages.

This research has some advantages, for instance, the relative large sample size and the subgroups analysis. However, there are several limitations in the present study. First of all, we diagnosed NAFLD using ultrasonography, which has limited in detecting mild liver steatosis. However, this noninvasive method is still widely used in clinical practice. Secondly, the study was cross-sectional in design, therefore, a causal relationship cannot be obtained. Thirdly, information about IR was unavailable in our study,which may be potential link.

\section{Conclusions}

TG/HDL-C is as effective as TyG in predicting NAFLD in Chinese men. TyG is superior to TG/HDL-C in identifying NFAFLD in Chinese overweight and obese women. While in Chinese normal and low weight women, TyG-HDL-C is a better predictor for NAFLD.

\section{Abbreviations}

AUC: area under the ROC curve; BMI: body mass index; DBP: diastolic blood pressure; FPG: fasting plasma glucose; HCY: homocysteine; HDL-C: high-density lipoprotein cholesterol; LDL-C: low-density 
lipoprotein cholesterol; NAFLD: nonalcoholic fatty liver disease; OR: odds ratio; ROC: receiver operator characteristic; SBP: systolic blood pressure; SUA: serum uric acid; TC: total cholesterol; TG: triglycerides; TG/HDL-C: TG to HDL ratio

\section{Declarations}

\section{Acknowledgements}

We thank all of the participants for their contribution to this study.

\section{Funding}

None.

\section{Availability of data and materials}

The datasets analyzed during the current study are available from the corresponding author on reasonable request.

\section{Authors' contributions}

Jinyan Ren and Fei Gao took part in study design and writing the manuscript. Xinfeng Wang and Yingmei Zheng collected the data. Hui Li and Xuan Yang performed the statistical analyses. All authors also read and approved the final version of this manuscript.

\section{Competing interests}

The authors declare that they have no competing interests.

\section{Consent for publication}

Not applicable.

\section{Ethics approval and consent to participate}

This study was approved by institutional review board of the Affiliated Hospital of Qingdao University, and the informed consent requirement was exempted because of our retrospective estimation of deidentified database.

\section{Author details}

${ }^{1}$ Health Management Center, the Affiliated Hospital of Qingdao University, 1677 Wutaishan Road, Qingdao 266555, China; ${ }^{2}$ Department of Hand and Foot Surgery, the Affiliated Hospital of Qingdao University, 1677 Wutaishan Road, Qingdao 266555, China. 


\section{References}

[1] Younossi ZM, Koenig AB, Abdelatif D, et al. Global epidemiology of nonalcoholic fatty liver diseaseMetaanalytic assessment of prevalence, incidence, and outcomes. Hepatology. 2016;64:73-84.

[2] Li Z, Xue J, Chen P. Prevalence of nonalcoholic fatty liver disease in mainland of China: a metaanalysis of published studies. J Gastroenterol Hepatol. 2014;29(1):42-51.

https://doi.org/10.1111/jgh.12428.

[3] Yki-Jarvinen H. Non-alcoholic fatty liver disease as a cause and a consequence of metabolic syndrome. Lancet Diabetes Endocrinol. 2014;2:901-10.

[4] Anstee QM, Targher G, Day CP. Progression of NAFLD to diabetes mellitus, cardiovascular disease or cirrhosis. Nat Rev Gastroenterol Hepatol. 2013;10:330-44.

[5] Stepanova M, Younossi ZM. Independent association between nonalcoholic fatty liver disease and cardiovascular disease in the US population. Clin Gastroenterol Hepatol. 2012;10:646-50.

[6] Lee JI, Kim MC, Moon BS, Song YS, Han EN, Lee HS, et al. The relationship between 10-year cardiovascular risk calculated using the pooled cohort equation and the severity of non-alcoholic fatty liver disease. Endocrinol Metab (Seoul). 2016;31:86-92.

[7] Targher G, Day CP, Bonora E. Risk of cardiovascular disease in patients with nonalcoholic fatty liver disease. N Engl J Med. 2010; 363: 1341-50.

[8] Zhang S, Du T, Zhang J, Lu H, Lin X, Xie J, Yang Y, Yu X. The triglyceride and glucose index (TyG) is an effective biomarker to identify nonalcoholic fatty liver disease. Lipids Health Dis. 2017 Jan 19;16(1):15. doi: 10.1186/s12944-017-0409-6. PMID: 28103934; PMCID: PMC5248473.

[9] Simental-Mendia LE, Simental-Mendia E, Rodriguez-Hernandez H, Rodriguez-

Moran M, Guerrero-Romero F. The product of triglycerides and glucose as biomarker for screening simple steatosis and NASH in asymptomatic women. Ann Hepatol. 2016;15:715-20.

[10] Fedchuk L, Nascimbeni F, Pais R, Charlotte F, Housset C, Ratziu V; LIDO Study Group. Performance and limitations of steatosis biomarkers in patients with nonalcoholic fatty liver disease. Aliment Pharmacol Ther. 2014 Nov;40(10):1209-22. doi: 10.1111/apt.12963. Epub 2014 Sep 29. PMID: 25267215.

[11] Fan N, Peng L, Xia Z, Zhang L, Song Z, Wang Y, Peng Y. Triglycerides to high-density lipoprotein cholesterol ratio as a surrogate for nonalcoholic fatty liver disease: a cross-sectional study. Lipids Health Dis. 2019 Feb 2;18(1):39. doi: 10.1186/s12944-019-0986-7. PMID: 30711017; PMCID: PMC6359827. 
[12] Fukuda Y, Hashimoto Y, Hamaguchi M, Fukuda T, Nakamura N, Ohbora A,Kato T, Kojima T, Fukui M. Triglycerides to high-density lipoprotein cholesterol ratio is an independent predictor of incident fatty liver; a population-based cohort study. Liver Int. 2016;36:713-20.

[13] Pacifico L, Bonci E, Andreoli G, Romaggioli S, Di Miscio R, Lombardo CV, Chiesa C. Association of serum triglyceride-to-HDL cholesterol ratio with carotid artery intima-media thickness, insulin resistance and nonalcoholic fatty liver disease in children and adolescents. Nutr Metab Cardiovasc Dis. 2014;24:737-43.

[14] Chen Z, Qin H, Qiu S, Chen G, Chen Y. Correlation of triglyceride to high-density lipoprotein cholesterol ratio with nonalcoholic fatty liver disease among the non-obese Chinese population with normal blood lipid levels: a retrospective cohort research. Lipids Health Dis. 2019 Aug 9;18(1):162. doi: 10.1186/s12944-019-1104-6. PMID: 31399032; PMCID: PMC6689160.

[15] Simental-Mendia LE, Rodriguez-Moran M, Guerrero-Romero F. The product of fasting glucose and triglycerides as surrogate for identifying insulin resistance in apparently healthy subjects. Metab Syndr Relat Disord. 2008;6:299-304.

[16] National Workshop on Fatty Liver and Alcoholic Liver Disease CSoH, Chinese Medical Association; Fatty Liver Expert Committee, Chinese Medical Doctor Association. Guidelines of prevention and treatment for nonalcoholic fatty liver disease: a 2018 update. Zhonghua Gan Zang Bing Za Zhi. 2018;26:195-203.

[17] Zheng R, Du Z, Wang M, Mao Y, Mao W. A longitudinal epidemiological study on the triglyceride and glucose index and the incident nonalcoholic fatty liver disease. Lipids Health Dis. 2018 Nov 20;17(1):262. doi: 10.1186/s12944-018-0913-3. PMID: 30458848; PMCID: PMC6247753.

[18] Buzzetti E, Pinzani M, Tsochatzis EA. The multiple-hit pathogenesis of non-

alcoholic fatty liver disease (NAFLD). Metabolism. 2016;65(8):1038-48.

https://doi.org/10.1016/j.metabol.2015.12.012.

[19] Birkenfeld AL, Shulman GI. Nonalcoholic fatty liver disease, hepatic insulin resistance, and type 2 diabetes. Hepatology. 2014;59:713-23.

[20] Machado M, Cortez-Pinto H. Non-alcoholic fatty liver disease and insulin resistance. Eur J Gastroenterol Hepatol. 2005;17:823-6.

[21] Samuel VT, Liu ZX, Qu X. Mechanism of hepatic insulin resistance in nonalcoholic fatty liver disease. J Biol Chem. 2004;279(31):32345-53. https://doi. org/10.1074/jbc.M313478200. 
[22] Kim JS, Kang HT, Shim JY, Lee HR. The association between the triglyceride to high-density lipoprotein cholesterol ratio with insulin resistance (HOMA-IR) in the general Korean population: based on the National Health and nutrition examination survey in 2007-2009. Diabetes Res Clin Pract. 2012;97:132-8.

[23] Zhou M, Zhu L, Cui X, Feng L, Zhao X, He S, Ping F, Li W, Li Y. The triglyceride to high-density lipoprotein cholesterol (TG/HDL-C) ratio as a predictor of insulin resistance but not of beta cell function in a Chinese population with different glucose tolerance status. Lipids Health Dis. 2016;15:104.

[24] Ren X, Chen ZA, Zheng S, Han T, Li Y, Liu W, Hu Y. Association between triglyceride to HDL-C ratio (TG/HDL-C) and insulin resistance in Chinese patients with newly diagnosed type 2 diabetes mellitus. PLoS One. 2016;11:e0154345.

[25] Pantoja-Torres B, Toro-Huamanchumo CJ, Urrunaga-Pastor D, Guarnizo-Poma M, Lazaro-Alcantara H, Paico-Palacios S, Del Carmen Ranilla-Seguin V, Benites-Zapata VA; Insulin Resistance and Metabolic Syndrome Research Group. High triglycerides to HDL-cholesterol ratio is associated with insulin resistance in normal-weight healthy adults. Diabetes Metab Syndr. 2019 Jan-Feb;13(1):382-388. doi: 10.1016/j.dsx.2018.10.006. Epub 2018 Oct 10. PMID: 30641729.

[26] Lee SH, Kwon HS, Park YM, et al. Predicting the development of diabetes using the product of triglycerides and glucose: the Chungju Metabolic Disease Cohort (CMC) study. PLoS ONE. 2014;9: e90430.

[27] Guerrero-Romero F, Simental-Mendia LE, Gonzalez-Ortiz M, Martinez- Abundis E, Ramos-Zavala MG, Hernandez-Gonzalez SO, et al. The product of triglycerides and glucose, a simple measure of insulin sensitivity. Comparison with the euglycemic-hyperinsulinemic clamp. J Clin Endocrinol Metab. 2010;95:3347-51.

[28] Vasques AC, Novaes FS, de Oliveira Mda S, Souza JR, Ya manaka A, Pareja JC, et al. TyG index performs better than HOMA in a Brazilian population: a hyperglycemic clamp validated study. Diabetes Res Clin Pract. 2011;93:e98-100.

[29] Du T, Yuan G, Zhang M, Zhou X, Sun X, Yu X. Clinical usefulness of lipid ratios, visceral adiposity indicators, and the triglycerides and glucose index as risk markers of insulin resistance. Cardiovasc Diabetol. 2014;13:146.

[30] Er LK, Wu S, Chou HH, Hsu LA, Teng MS, Sun YC, Ko YL. Triglyceride Glucose-Body Mass Index Is a Simple and Clinically Useful Surrogate Marker for Insulin Resistance in Nondiabetic Individuals. PLoS One. 2016 Mar 1; 11(3): e0149731.

[31] Babic N, Valjevac A, Zaciragic A, Avdagic N, Zukic S, Hasic S. The Triglyceride/HDL Ratio and Triglyceride Glucose Index as Predictors of Glycemic Control in Patients with Diabetes Mellitus Type 2. 
Med Arch. 2019 Jun;73(3):163-168. doi: 10.5455/medarh.2019.73.163-168. PMID: 31404127; PMCID: PMC6643328.

[32] Ren X, Chen ZA, Zheng S, et al. Association between Triglyceride to HDL-C Ratio (TG/HDL-C) and Insulin Resistance in Chinese Patients with Newly Diagnosed Type 2 Diabetes Mellitus. PLoS One. 2016;11:e0154345.

[33] Ren XY, Shi D, Ding J, Cheng ZY, Li HY, Li JS, Pu HQ, Yang AM, He CL, Zhang JP, Ma YB, Zhang YW, Zheng TZ, Bai YN, Cheng N. Total cholesterol to high-density lipoprotein cholesterol ratio is a significant predictor of nonalcoholic fatty liver: Jinchang cohort study. Lipids Health Dis. 2019 Feb 11;18(1):47. doi: 10.1186/s12944-019-0984-9. PMID: 30744645; PMCID: PMC6371543.

[34] Souza MR, Diniz Mde F, Medeiros-Filho JE, Araujo MS. Metabolic syndrome and risk factors for nonalcoholic fatty liver disease. Arq Gastroenterol. 2012 Jan-Mar;49(1):89-96. doi: 10.1590/s000428032012000100015 . PMID: 22481692.

[35] Zhang M, Deng Q, Wang L, Huang Z, Zhou M, Li Y, Zhao Z, Zhang Y, Wang L. Prevalence of dyslipidemia and achievement of low-density lipoprotein cholesterol targets in Chinese adults: A nationally representative survey of 163,641 adults. Int J Cardiol. 2018 Jun 1;260:196-203. doi: 10.1016/j.ijcard.2017.12.069. Erratum in: Int J Cardiol. 2018 May 12;: PMID: 29622441.

\section{Figures}
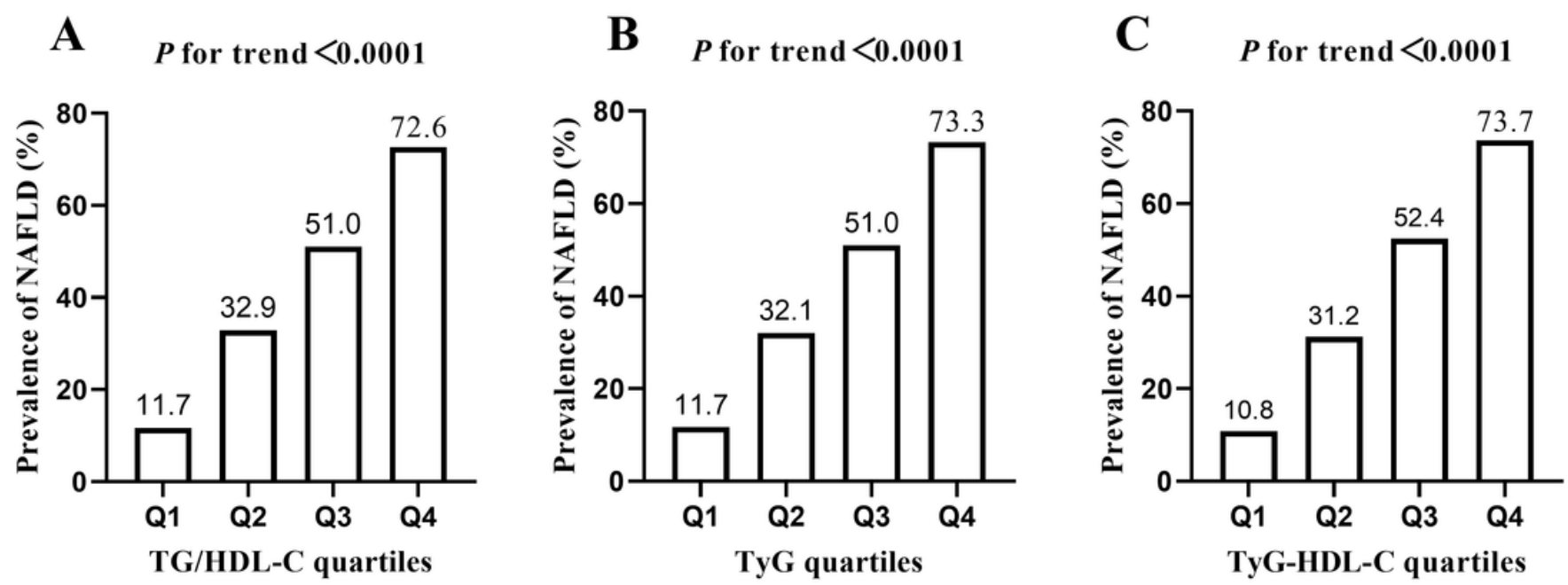

Figure 1

Prevalence of NAFLD according to the quartiles of TG/HDL-C (A), TyG(B) or TyG-HDL-C (C). Classification of TG/HDL-C quartiles: Q1 ( 0.57), Q2 (0.58 0.90), Q3 (0.91 1.48), Q4 (1.49 ); TyG quartiles: Q1 ( 8.17), Q2 (8.18 8.59), Q3 (8.60 9.04), Q4 (9.05 ); TyG-HDL-C quartiles: Q1 ( 6.59), Q2 (6.60 7.16), 
Q3 (7.17 7.73), Q4 (7.74 ). NAFLD, nonalcoholic fatty liver disease; Q1, first quartile; Q2, second quartile; Q3,third quartile; Q4, fourth quartile
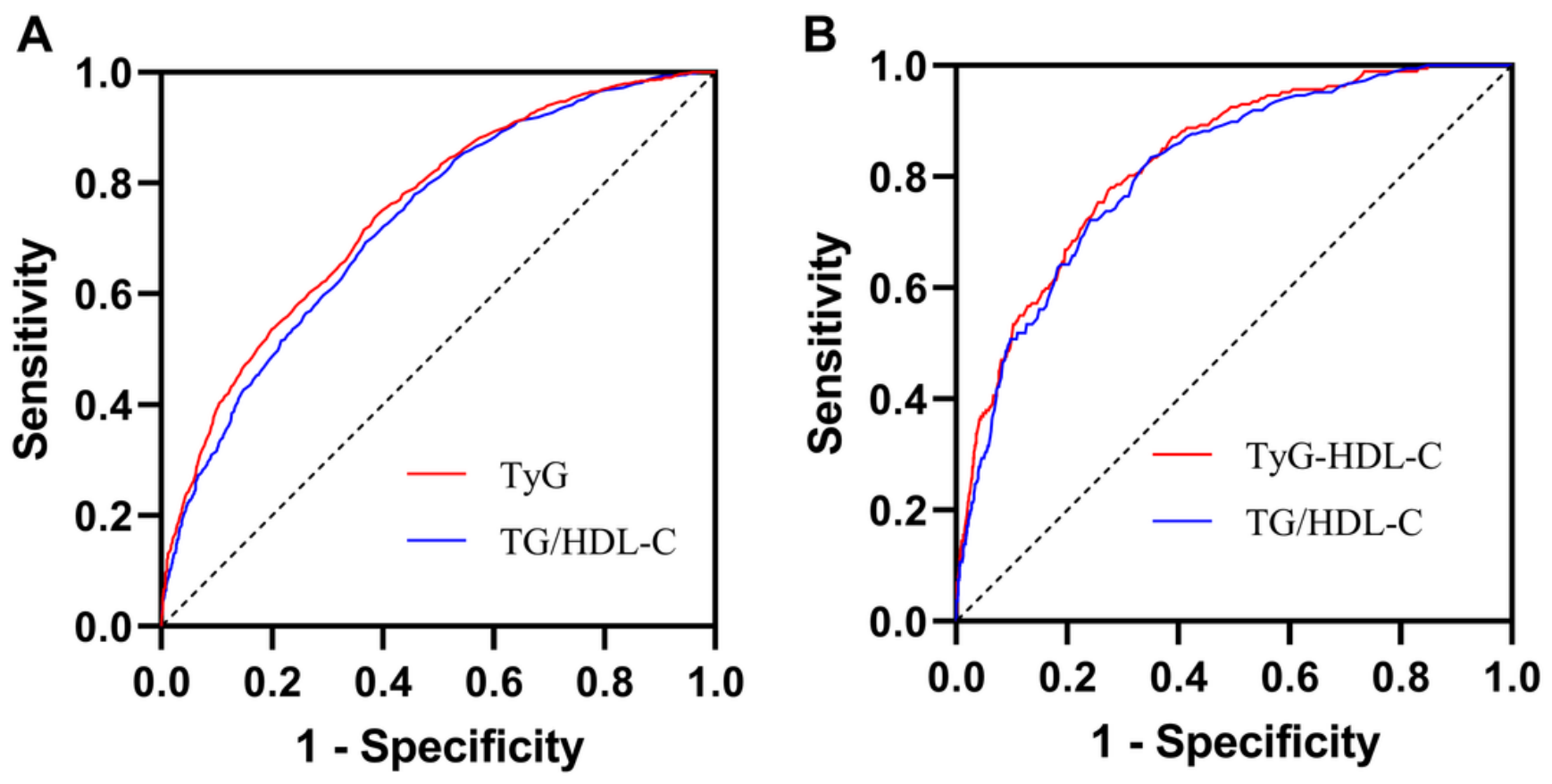

Figure 2

ROC curves for NAFLD in women. A ROC curves in overweight and obese women. The AUC of TyG and TG/HDL-C were 0.748 (95\% Cl 0.732-0.763), 0.724 (95\% Cl 0.712-0.744), respectively. P value for the difference was $<0.0001$. B ROC curves in normal and low weight women. The AUC of TyG-HDL-C, and TG/HDL-C were 0.827 (95\% Cl 0.810-0.843), 0.813 (95\% Cl 0.796-0.830), respectively. $P$ value for the difference was $=0.0015$. 